Supporting information for

Viscoelastic fluid formed by ultra-long-chain erucic acid-base ionic liquid surfactant responds to acid/alkaline, $\mathrm{CO}_{2}$ and light

Zhe Yang, ${ }^{a}$ Shuai He, ${ }^{b}$ Yinjun Fang, ${ }^{c}$ Yongmin Zhang ${ }^{a}{ }^{*}$

${ }^{a}$ Key Laboratory of Synthetic and Biological Colloids, Ministry of Education, School of Chemical \& Materials Engineering, Jiangnan University, No.1800 Lihu Avenue, Wuxi 214122, P. R. China

${ }^{\mathrm{b}}$ College of Chemistry and Environmental Protection Engineering, Southwest Minzu University, Chengdu 610041, P. R. China

${ }^{\mathrm{c}}$ Zanyu Technology Group Company Ltd., Hangzhou 310009, P. R. China

Corresponding author: Y.M. Zhang, E-mail: zhangym@jiangnan.edu.cn

\title{
Contents
}

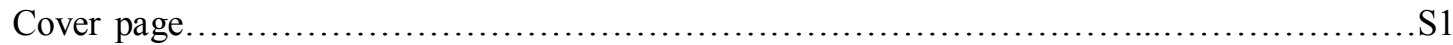

Figure S1 1H NMR spectrum of benzyltrimethyl ammonium erucate (ErBTA) ...................S2

Figure S2 ${ }^{13} \mathrm{C}$ NMR spectrum of benzyltrimethyl ammonium erucate (ErBTA) ..................S2

Figure S3 FT-IR spectra of benzyltrimethyl ammonium hydroxide (BTAOH), erucic acid, ErBTA

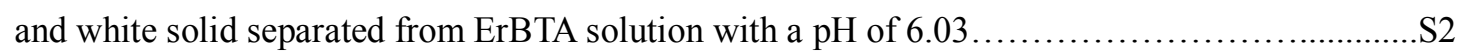

Figure S4 Diff erential scanning calorimetry thermogram of ErBTA..........................S3

Figure S5 Snapshots of $1 \mathrm{wt} \%$ ErBTA solution under different temperature......................S3

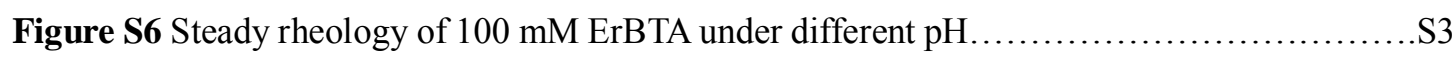

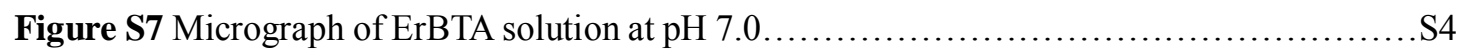

Figure S8 Steady rheology of $100 \mathrm{mM}$ ErBTA under different exposure to $\mathrm{CO}_{2} \ldots \ldots \ldots \ldots \ldots \ldots$. . . 4

Figure S9 Snapshots of binary solution of ErBTA/ DPIN (100/15 mmol/L) at different exposure

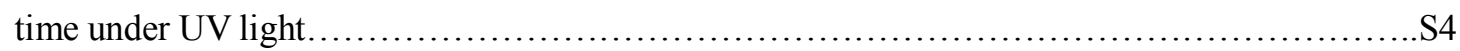

Figure S10 Variations in flow curves of choline erucate with concentration..................S5 


\section{Additional results}

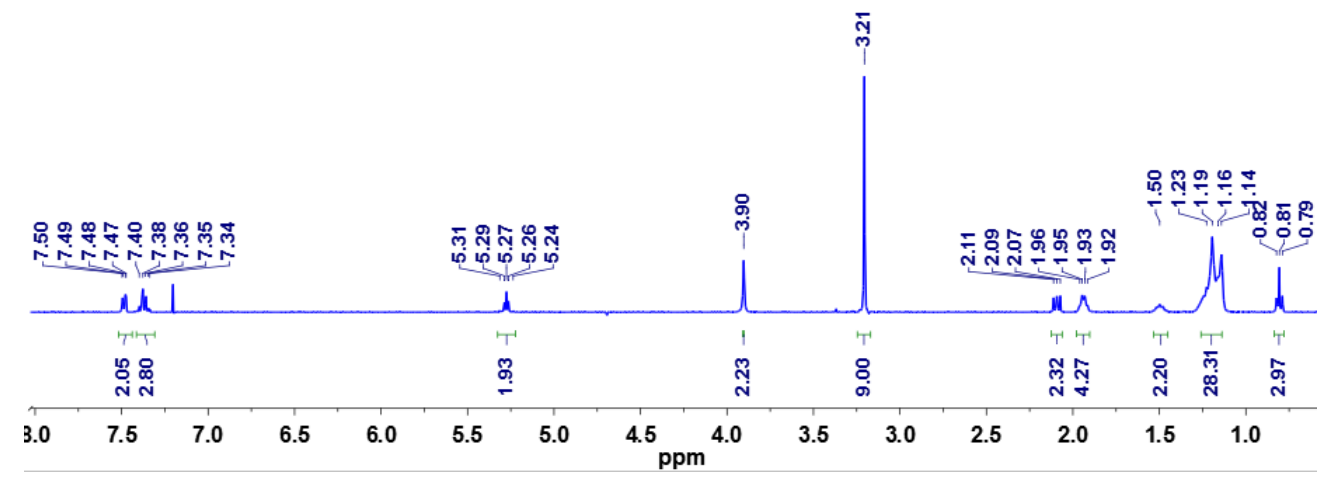

Figure S1 ${ }^{1} \mathrm{H}$ NMR spectrum of benzyltrimethyl ammonium erucate (ErBTA).

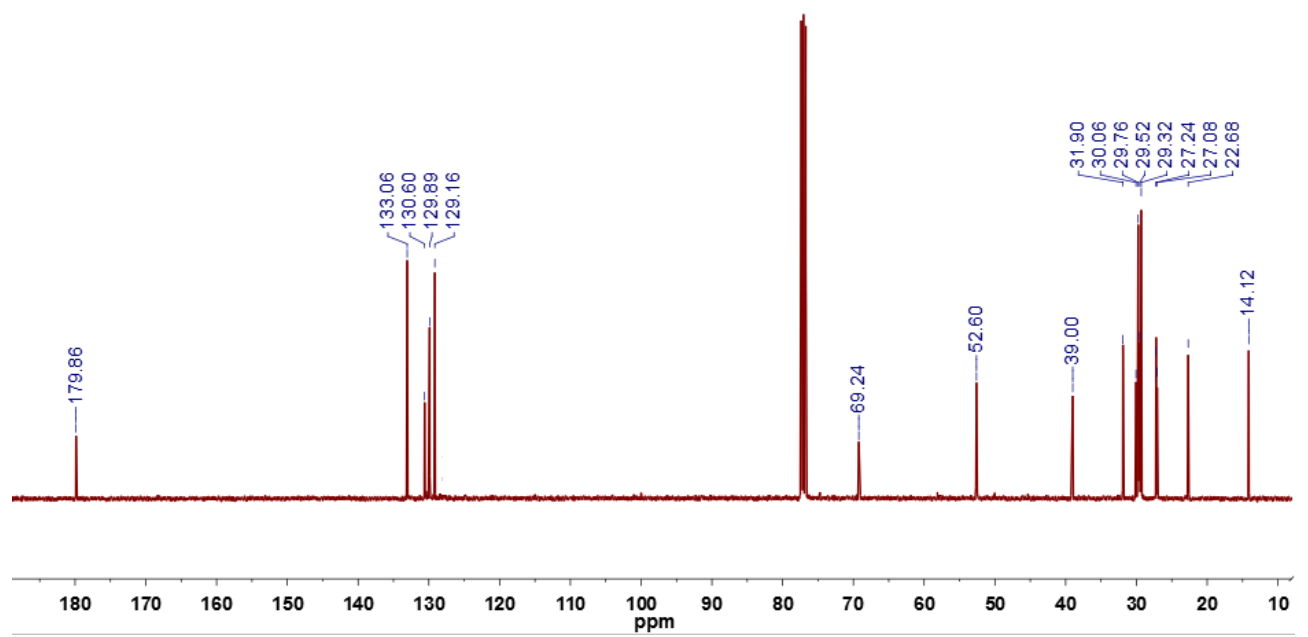

Figure S2 ${ }^{13} \mathrm{C}$ NMR spectrum of benzyltrimethyl ammonium erucate (ErBTA).

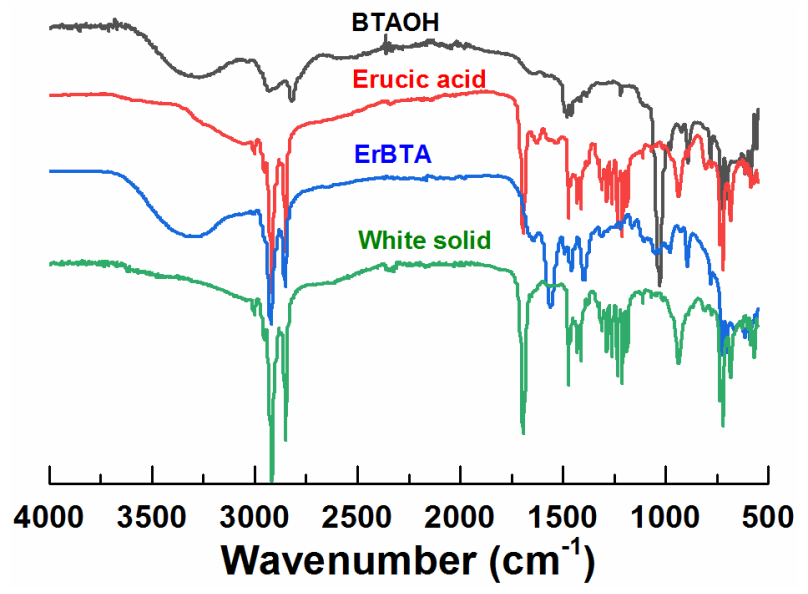

Figure S3 FT-IR spectra of benzyltrimethyl ammonium hydroxide (BTAOH), erucic acid, ErBTA and white solid separated from ErBTA solution with a pH of 6.03. 


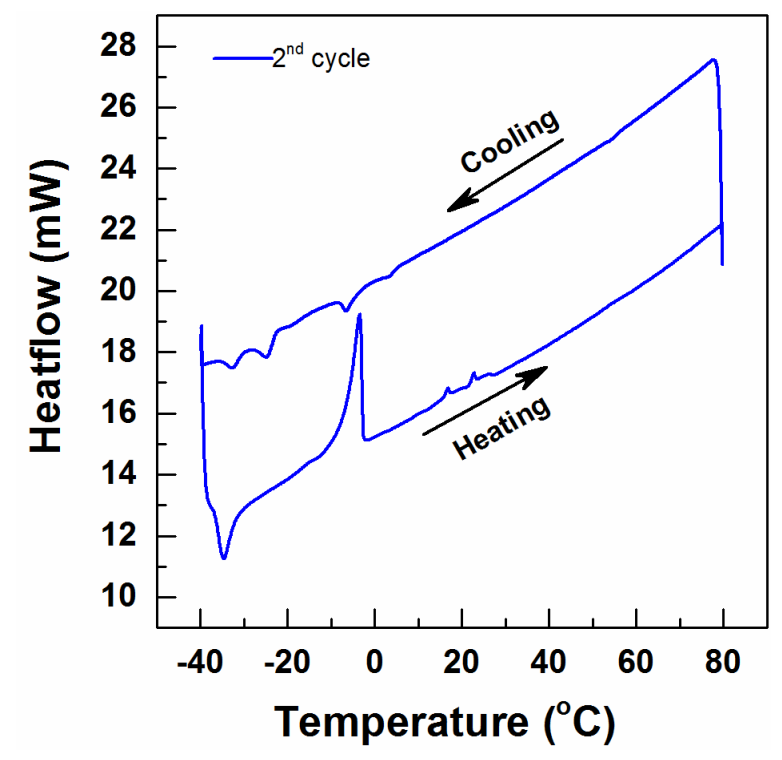

Figure S4 Differential scanning calorimetry thermogram of ErBTA.

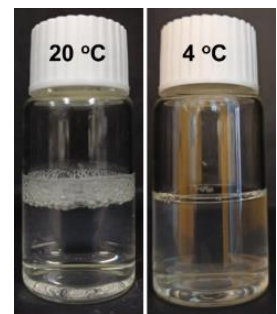

Figure S5 Snapshots of 1 wt \% ErBTA solution under different temperature.

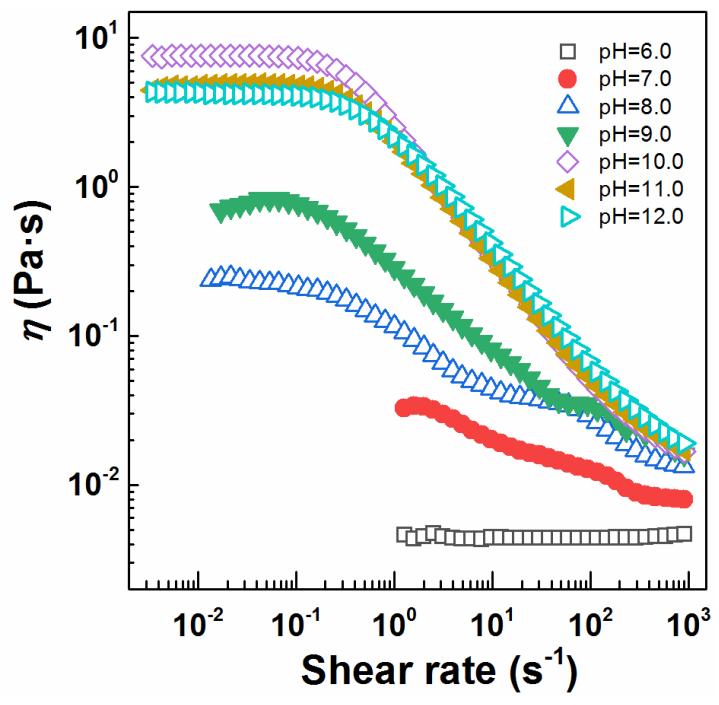

Figure S6 Steady rheology of 100 mM ErBTA under different pH. 
Figure S7 Micrograph of ErBTA solution at pH 7.0.

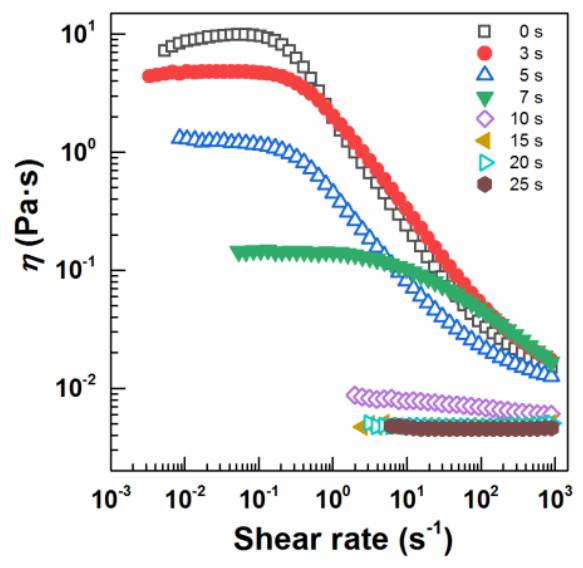

Figure S8 Steady rheology of $100 \mathrm{mM}$ ErBTA under different exposure to $\mathrm{CO}_{2}$.

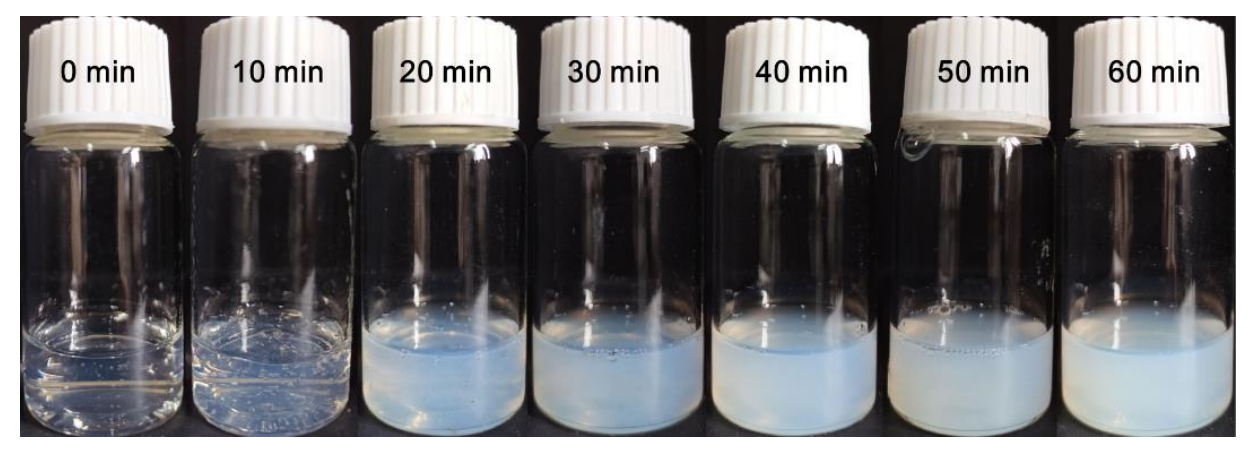

Figure S9 Snapshots of binary solution of ErBTA/ DPIN (100/15 mmol/L) at different exposure time under UV light. 


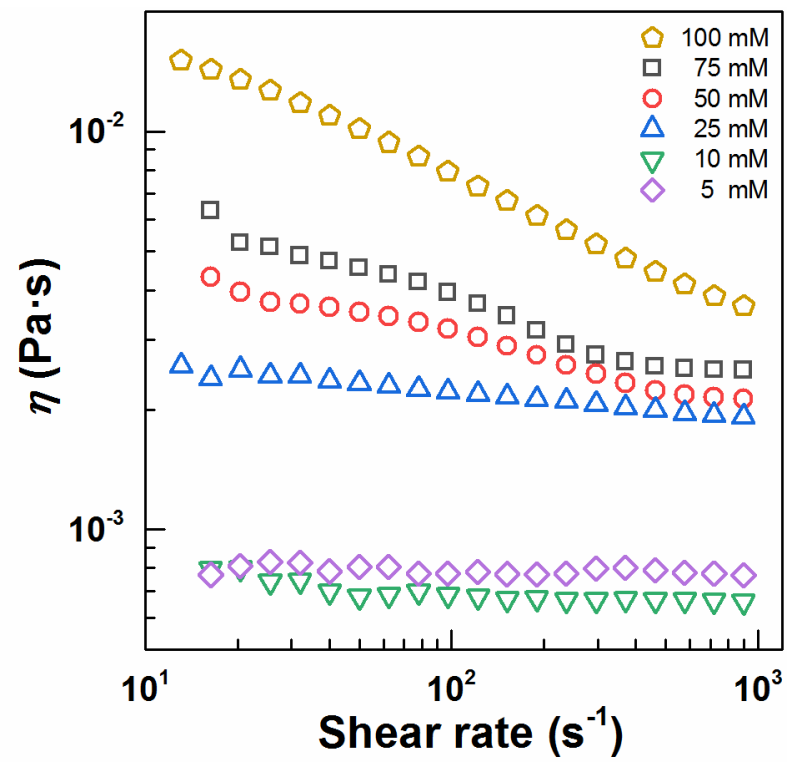

Figure S10 Variations in flow curves of choline erucate with concentration. 